\title{
Malabsorción de lactosa en pacientes con enfermedad inflamatoria intestinal inactiva: ¿está justificado excluir los productos lácteos a todos los pacientes?
}

\author{
R. BAÑOS MADRID, H. SALAMA BENARROCH, S. MORÁN SÁNCHEZ, F. \\ GALLARDO SÁNCHEZ, A. ALBALADEJO MEROÑO, J. MERCADER MARTÍNEZ
}

Servicio de Aparato Digestivo. Hospital Universitario Virgen de la Arrixaca. Murcia

\begin{abstract}
LACTOSE MALABSORPTION IN PATIENTS WITH INFLAMMATORY BOWEL DISEASE WITHOUT ACTIVITY: IT WOULD BE NECESSARY TO EXCLUDE THE LACTOSE PRODUCTS IN THEIR DIET TO ALL THE PATIENTS?
\end{abstract}

\section{RESUMEN}

Introducción: Muchos pacientes con enfermedad inflamatoria intestinal (EII) sin evidencia de malabsorción de lactosa, evitan tomar productos lácteos, basándose en percepciones erróneas y consejos equivocados de médicos y libros de dietética.

Objetivo: Evaluar la frecuencia de malabsorción de lactosa en pacientes EII.

Método: Se estudia la frecuencia de intolerancia a la lactosa en 18 pacientes con enfermedad de Crohn, 24 pacientes con colitis ulcerosa y 25 controles sanos, realizando un test de hidrógeno espirado.

Resultados: Se observaron deficiencias de absorción de la lactosa en $7(16,6 \%)$ pacientes con EII y $5(20 \%)$ sujetos control.

Conclusiones: No todos los enfermos con EII son intolerantes a lactosa, por lo que no debe generalizarse la supresión de los lácteos en la dieta de estos enfermos.

PALABRAS CLAVE: Enfermedad inflamatoria intestinal. Malabsorción de lactosa. Test de aliento.

\section{ABSTRACT}

Introduction: There are inflammatory bowel disease (IBD) patients avoid lacteal products without evidence of lactose malabsorption, probably because of incorrect patient perceptions and arbitrary advice from physicians and diet books.

Aim: To evaluate the prevalence of lactose malabsorption in patients with IBP.

Method: In 18 patients with Crohn's disease and 24 with ulcerative colitis and 25 controls the prevalence of lactose intolerance, as measured by lactose breath hydrogen tests.

Results: Observed deficiencies of absorption of the lactose in 7 (16.6\%) patients with IBP and 5 (20\%) subjects control.

Conclusions: Not all the patients with IBD are intolerant to the lactose by the suppression of the lacteal produts must not be generalized in the diet of these patients.

KEY WORDS: Inflammatory bowel disease. Lactose malabsorption. Breath testing.

Baños Madrid R, Salama Benarroch H, Morán Sánchez, S, Gallardo Sánchez, F, Albaladejo Meroño A, Mercader Martínez J. Malabsorción de lactosa en pacientes con enfermedad inflamatoria intestinal inactiva: ¿está justificado excluir los productos lácteos a todos los pacientes? An Med Interna (Madrid) 2004; 21: 212-214.

\section{INTRODUCCIÓN}

En muchas ocasiones los pacientes con (EII) consumen menos leche de la que pueden consumir, ya sea por consejos erróneos de su médico o por decisión propia, favoreciendo el desarrollo de enfermedades óseas metabólicas por baja absorción de calcio, sobre todo en pacientes que toman corticoesteroides durante largos períodos de tiempo. No hay estudios que demuestren que la leche sea mala para todos los pacientes con EII $(1,2)$. Se suele retirar durante los brotes, pero durante la remisión la leche es un alimento completo que contiene mucho calcio, así un litro de leche al día es suficiente para combatir la osteoporosis (3).

La finalidad de este estudio es valorar la frecuencia de aparición de intolerancia a la lactosa en pacientes con EII inactiva.

\section{MATERIAL Y MÉTODOS}

Se realizó el estudio a 42 pacientes con EII que acudieron a revisión en consulta, sin brote en el momento de realizar la prueba, la relación hombres/mujeres era 0,9/1 con una edad media de 38,5 años (rango: 16-72 años), de los cuales 24 estaban afectos de colitis ulcerosa (CU), 18 de enfermedad de Crohn (EC) y a 25 controles sanos con una relación hombres/mujeres de 1,1/1 y una media de edad de 34 años (rango: 19-65 años). La media de años de evolución de la enfermedad fue de 5,3 años (1-23 años) (Tabla I).

Se utiliza un monitor portátil de hidrógeno (H2) espirado (Fig. 1) con el fin de detectar deficiencias en la absorción de lactosa. Como protocolo de realización del test (Tabla II) se recoge una muestra alveolar para establecer un valor basal de

Trabajo aceptado: 16 de diciembre de 2003 
TABLA I

\begin{tabular}{lll}
\multicolumn{3}{c}{ CARACTERÍSTICAS DE LOS GRUPOS } \\
\hline Grupo & Ell & Control \\
\hline (n=67) & $42(24$ CU/18 EC) & 25 \\
Edad (media +/- DE), años & $38,5+/-11,7$ & $34+/-14,8$ \\
Relación hombres/mujeres & $0,9 / 1$ & $1,1 / 1$ \\
\hline
\end{tabular}

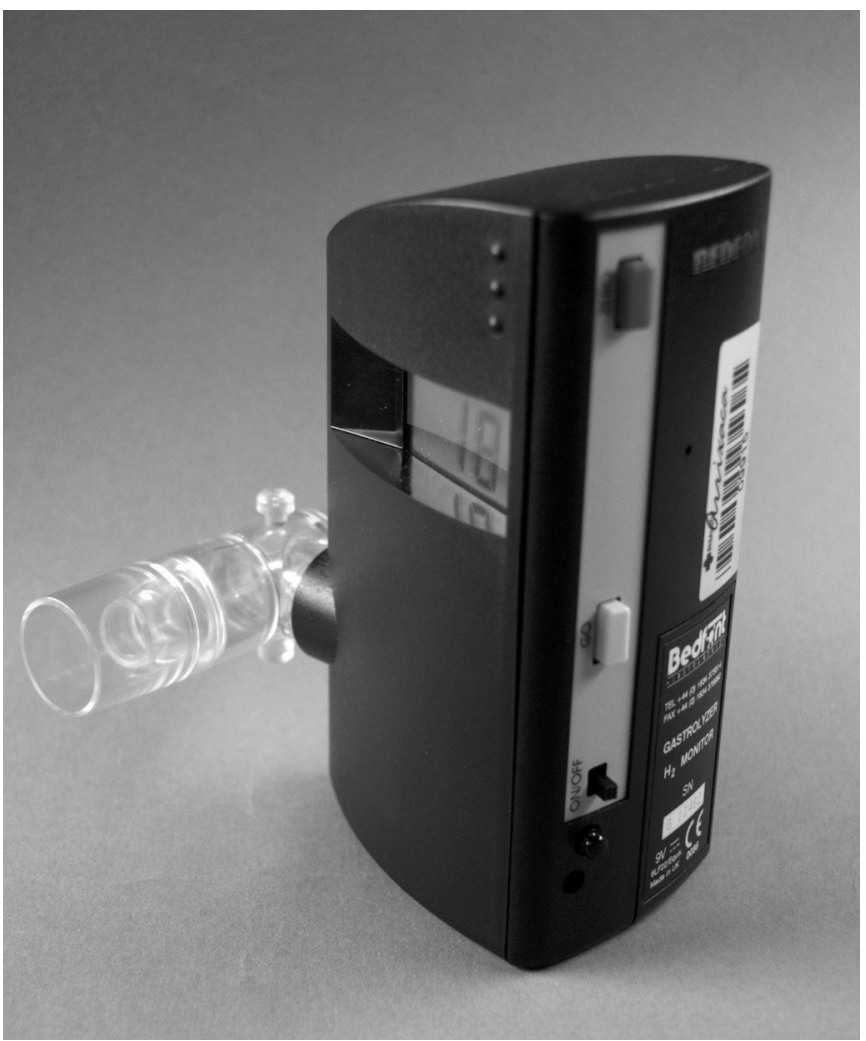

Fig. 1. Monitor portátil de hidrógeno espirado.

\section{TABLA II}

PROTOCOLO DE ACTUACIÓN (TEST DE INTOLERANCIA A LA LACTOSA)

1. Instrucciones generales para test con $\mathrm{H} 2$

2. Recoger la muestra en ayunas

3. Muestra basal

4. Tomar 25 gr de lactosa

5. Recoger muestras cada 30 minutos empezando 1 hora después de la toma, hasta tres horas

6. Resultado positivo sí $\mathrm{H} 2>$ ó $=$ a 20 ppm + basal

H2, que debe ser inferior a 10 partes por millón (p.p.m.). Valores más altos pueden indicar ayuno incompleto antes de la prueba (el ayuno debe ser de 12 horas como mínimo), ingestión de comida de lenta digestión antes de la prueba, o si el valor basal excede de 20 ppm la presencia de sobrecrecimiento bacteriano. Como dosis de lactosa se usa $25 \mathrm{~g}$ para cada paciente y control sano. Posteriormente se recoge una muestra alveolar y se analiza cada 30 minutos empezando una hora después de la toma de la lactosa hasta tres horas. Se considera la prueba positiva si aparecían síntomas o se producía un aumento de más de 20 ppm sobre la toma basal. Además, se realizó una prueba de aliento de lactulosa cuando no se observó excreción de $\mathrm{H} 2$ para detectar individuos no productores de H2. Se excluyeron del estudio aquellos sujetos con EII activa (índice de actividad CDAI $>150$ para la EC /índice de actividad de Truelove-Witts en la CU) o que habían tomado recientemente antibióticos, enemas, laxantes o antidiarréicos, así como pacientes con historial de EPOC.

El análisis de los datos fue realizado con el programa estadístico SPSS/Windows 8.0. Los resultados de los grupos se expresan en porcentajes y media aritmética con desviación estándar. En la comparación de medias se empleó la t de Student. El nivel de significación se estableció para valores de $\mathrm{p}<0,05$.

\section{RESULTADOS}

Se observaron deficiencias de absorción de la lactosa o intolerancia en 7 pacientes con EII (16,6 \%), (3 con EC y 4 con CU) y 5 sujetos control (20\%) (Fig. 2). La diferencia con respecto a la intolerancia a la lactosa entre el grupo control y el grupo con EII resultó estadísticamente no significativa. Todos los positivos en el test de $\mathrm{H} 2$ espirado presentaban intolerancia a la leche entera con sintomatología florida tras la toma de leche entera (Tabla III). No se registró ningún paciente con sobrecrecimiento bacteriano ni deficiencia de producción de H2. Todos los pacientes consumían productos lácteos antes del diagnóstico de EII, pero el 59,5 \% (25 pacientes con EII) excluyó estos productos de su dieta después del diagnóstico, haciéndolo la mayoría (20 pacientes con EII) por prescripción médica $(80 \%)$.

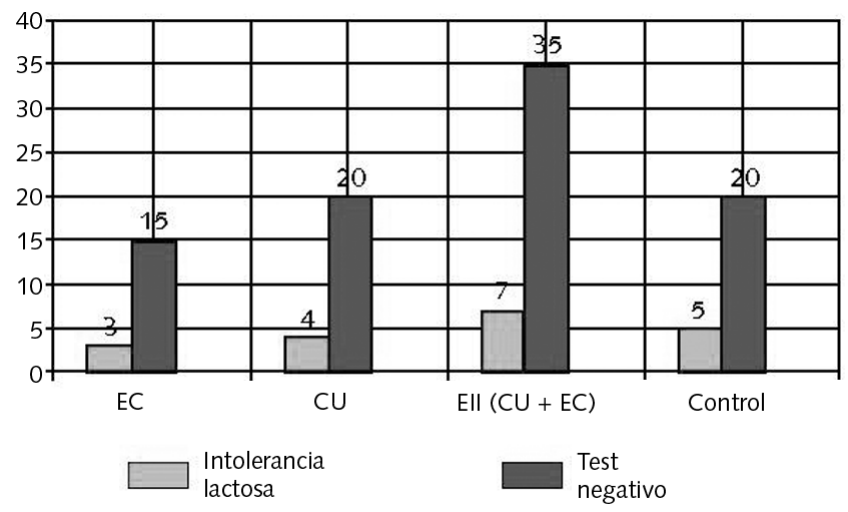

Fig. 2. Resultados test del aliento

\section{DISCUSIÓN}

En Europa, la incidencia de intolerancia a la lactosa tardía oscila entre el 0 y el $20 \%$ en las distintas publicaciones. La intolerancia a la lactosa es la incapacidad de digerir cantidades significativas de lactosa que provoca una serie de sínto- 


\section{TABLA III}

CUESTIONARIO DE VALORACIÓN DE TOLERANCIA A LA LECHE

1. Refiere algunos de estos síntomas tras la toma de leche:

Dolor abdominal (3)

Distensión abdominal (3)

Náuseas (1)

Vómitos

Eructos

Flatulencia (1)

Borborigmos

Diarrea (2)

2. Desaparición de estos síntomas tras la retirada de la leche

Se consideraba que presentaba intolerancia cuando cumplía alguno de los criterios del punto 1 y el punto 2 .

Entre paréntesis el número de pacientes que presentaba ese síntoma en nuestro estudio.

mas como son dolor abdominal, nauseas, distensión abdominal, flatulencias, borborigmos y diarrea, pudiendo aparecer pérdida de peso, con malnutrición. Dichos síntomas comienzan alrededor de 30 minutos a 2 horas después de comer o beber alimentos que contienen lactosa (4).

Esta incapacidad se debe a la carencia en mayor o menor grado de B-galactosidasa (lactasa), un enzima producido por las células de la pared del intestino delgado. El grado de severidad de los síntomas depende de la cantidad de lactosa que cada individuo tolera. El diagnostico definitivo requiere la demostración de la baja actividad de la lactosa en una muestra en la biopsia intestinal. Debido a la dificultad de obtener estas muestras, se han utilizado métodos indirectos validados para esta valoración. Aunque no todas las personas con esta deficiencia tienen síntomas, aquellas que lo sufren son consideradas como intolerantes a la lactosa.

Son múltiples los factores o las situaciones que se han implicado en el desarrollo de la osteoporosis: genéticos, sexo femenino, menarquia tardía, menopausia precoz, tabaco, sedentarismo o el consumo de alcohol. Existen algunos de especial relevancia en los pacientes con EII, como el consumo bajo de lácteos, el uso de esteroides en el tratamiento de los brotes de actividad y la malnutrición asociada a la enfermedad (3).

La restricción de productos lácteos en la dieta es una medida habitual, bien por prescripción médica o por la asociación que hacen los pacientes con EII de determinados síntomas como son (diarrea, dolor abdominal, distensión abdominal o nauseas) con la ingesta de lácteos. En la actualidad no hay ningún estudio que haya demostrado ningún efecto deletéreo de los lácteos sobre la EII. Tampoco se ha demostrado una mayor prevalencia de intolerancia y/o malabsorción a la lactosa con respecto a la población general. Sin embargo, una dieta pobre o exenta de productos lácteos podría ser perjudicial al suprimir la principal fuente de calcio y, por ello, favorecer la pérdida de masa ósea (3). Por lo tanto, en ausencia de datos que sugieran intolerancia a la lactosa, no parece recomendable excluir los lácteos de la dieta de estos pacientes; incluso en casos de intolerancia a la leche es habitual la tolerancia de otros productos lácteos como el yogur. Aquellos sujetos con intolerancia o deficiencias de absorción de la leche absorben y toleran bien el yogur $(1,5)$.

La supresión de lácteos sólo estaría indicado, en aquellos pacientes en los que se evidenciaria una intolerancia a la lactosa. La prevalencia en nuestro medio resultó similar a la de otras series publicadas (12-30\% de la población).

El hidrógeno $(\mathrm{H} 2)$ se genera en el interior del intestino, por la acción bacteriana sobre los carbohidratos en el intestino grueso y delgado. $\mathrm{El} \mathrm{H} 2$ resultante se difunde por el sistema circulatorio y de allí a los alveolos, después puede ser detectado en el aire espirado. Levitt(1969) demostró la correlación entre la producción de $\mathrm{H} 2$ en el interior de los intestinos y la secreción de $\mathrm{H} 2$ en el aire espirado. Así, la precisa medición del H2 en partes por millón en el aire espirado, revela la descomposición anormal y/o mala absorción de los carbohidratos (6).

La utilización de este tipo de equipos para el diagnostico de deficiencias en la absorción de la lactosa, tiene una serie de ventajas sobre los métodos convencionales bioquímicos, no es invasivo, es fácil de usar y proporciona resultados inmediatos. Su uso no se limita solo a la detección de la malabsorción de lactosa, permite la detección de la mal absorción de otros carbohidratos, diagnostico del sobrecrecimiento bacteriano y determinación de tránsito intestinal (6).

Así pues se puede concluir que no todos los enfermos con EII son intolerantes a la lactosa, por lo que no debe generalizarse la supresión de los lácteos en la dieta de estos enfermos, ya que es la principal fuente de calcio.

\section{Bibliografía}

1. Mishkin S. Dairy sensitivity, lactose malabsorption, and elimination diets in inflammatory bowel disease. Am J Clin 1997; 65: 464-7.

2. Kirschner BS, DeFavaro MV, Jensen W. Lactose malabsorption in children and adolescents with inflammatory bowel disease. Gastroenterology 1981; 81: 829-32.

3. Dinca M, Fries W, Luisetto G, Peccolo F, Bottega F, Leone L, et al. Evolution of osteopenia in inflammatory bowel disease. Am J Gastroenterol

1999; 94: 1292-1297.

4. Shaw AD, Davies GJ. Lactose intolerance: problems in diagnosis and treatment. J Clin Gastroenterol 1999; 28: 208-216.

5. Bernstein CN, Ament M, Artinian L, Ridgeway J, Shanahan F. Milk tolerance in adults ulcerative colitis. Am J Gastroenterol 1994; 89: 872-877.

6. Fleming, SC. Evaluation of hand-held Hydrogen monitor in the diagnosis of intestinal lactose deficiency. Ann Clin Biochem 1990; 27: 499-500. 